\title{
EDITORIAL
}

\section{Pharmacogenetics and mental disease}

As the array of potent behaviour-modifying drugs has grown, physicians employing these agents have become increasingly aware of the striking differences among individuals in therapeutic effectiveness and side-effects of the drugs (Omenn and Motulsky, 1973). These differences may be caused by different rates of biotransformation or elimination of the pharmacologically active species, different susceptibility to the drug action on specific cell receptors, or different mechanisms underlying a common psychiatric syndrome.

\section{PHARMACOGENETICS OF SPECIFIC DRUGS}

General discussion of the pharmacogenetics of specific drugs may be found in Kalow (1962), Motulsky (1964), Evans (1969), and Omenn and Motulsky (1973).

SUCCINYL CHOLINE (SUXAMETHONIUM) This depolarizing muscle relaxant is used widely in premedication for anaesthesia and for electroconvulsive therapy, because of its rapid onset and short duration of action. However, suxamethonium will paralyse breathing for several hours in the one in 2,500 Caucasians who has an abnormal form of the plasma enzyme pseudocholinesterase. A perfectly normal individual is genetically susceptible to a drúg-induced catastrophe because the enzyme required to inactivate the drug does not function properly. In the absence of the drug, there are no known abnormalities. The psychiatrist or anaesthetist should inquire about personal or family history of sensitivity to suxamethonium; a simple screening test is available (Morrow and Motulsky, 1968). Equipment for sustained respiratory assistance should be at hand.

ACETYLATION IN THE LIVER The antituberculosis agent isoniazid (INH) and a number of other drugs are inactivated in the liver by an acetylating enzyme. Population screening shows that about $50 \%$ of Caucasians and Negroes and $15 \%$ of Orientals are 'slow acetylators', due to less activity of the enzyme; family studies prove that the trait is determined by a single gene. Since slow acetylators develop higher blood levels of drug on a standard dose, peripheral neuropathy from INH, CNS, and gastrointestinal symptoms from the antidepressant phenelzine (Nardil) (Evans et al., 1965), and a lupus-like syndrome from hydralazine (Apresoline) occur in slow acetylators and not in rapid acetylators. Greater therapeutic effectiveness of phenelzine was reported for patients with neurotic depression who were slow acetylators (Johnstone and Marsh, 1973). Slow acetylators taking INH also are much more sensitive to toxicity from phenytoin (Dilantin), because INH in high concentrations inhibits metabolism of phenytoin and other drugs (Kutt, 1971).

OXIDANT DRUGS AND G6PD DEFICIENCY Glucose-6-phosphate dehydrogenase (G6PD) is the first enzyme of the energy-generating pentose-phosphate shunt pathway, essential to maintaining the integrity of the red blood cell. Deficiency of G6PD occurs with significant frequency in many population groups originating in subtropical and tropical countries, such as Africans, Southeast Asians, Indians, and Mediterraneans. Many drugs can precipitate acute haemolytic anaemia in these otherwise healthy but genetically predisposed individuals (Motulsky, 1972). The drug does not interact directly with the abnormal enzyme; rather, the red cells are more susceptible to drug injury. Several different mutations affecting G6PD cause enzyme deficiency. The Mediterraneantype G6PD deficiency is more severe than the Negro type, hence a larger number of drugs are a 
threat (Motulsky, 1972). Haemolytic anaemia caused by eating fava or broad beans (favism) occurs only in G6PD-deficient persons.

TRICYCLIC ANTIDEPRESSANTS: NORTRIPTYLINE The likelihood of side-effects from nortriptyline is correlated with the plasma concentration of the drug and not with the dose administered. Twin studies and family studies indicate a major role for genetic factors in the rate of elimination of nortriptyline, but the biochemical basis for individual differences is not yet known (Alexanderson and Sjöqvist, 1971).

\section{PHARMACOGENETICS IN SPECIFIC MENTAL DISORDERS}

DEPRESSION (AFFECTIVE DISORDERS) There is considerable evidence from twin and family studies that depressions (especially manic-depressive psychosis) are conditioned by genetic factors (Gershon et al., 1971). According to the biogenic amine hypothesis (Schildkraut, 1969), depression appears to be associated with decreased action or turnover of noradrenaline (NE) and serotonin, while manic states are associated with increased biogenic amine turnover. Pharmacogenetic agents which deplete NE from nerve terminals (reserpine) or interfere with its biosynthesis (alpha-methyl tyrosine, alpha-methyl dopa) may precipitate depression. Drugs which enhance biosynthesis of NE (L-dopa) may induce hypomanic states, and agents which prolong the action of NE by inhibiting intraneuronal monoamine oxidase (MAO inhibitors) or the neuronal re-uptake of NE released into the synapse (tricyclics) are effective antidepressants. Electroconvulsive shock also acts to increase tyrosine hydroxylase activity and NE turnover (Musacchio et al., 1969). Clinical observations suggest likely pharmacogenetic relationships among patients with depression. Thus, Pare et al. (1962) reported that two groups of patients could be differentiated by their response to either MAO inhibitors or tricyclic compounds (Pare et al., 1962; Pare and Mack, 1971). In their experience, patients who responded to one class of antidepressant tended not to respond to the other. Patients showed the same pattern of pharmacological responsiveness during a subsequent episode of depression, which might have been precipitated by quite different life stresses. Also, relatives who had affective disorders shared the pattern of pharmacological responsiveness or unresponsiveness. Angst obtained comparable results with imipramine in family studies (Angst, 1964). While additional studies with placebo controls are required to substantiate these findings, it is easy to conceive of variations at each of the steps in biogenic amine biosynthesis and metabolism that would produce individual differences in the effectiveness of drugs in ameliorating or inducing affective disorders (Omenn, 1973a). About 10\% of the patients treated with reserpine will develop depression (Harris, 1957). These patients have a history of affective disorder more often than do the $90 \%$ of reserpine-treated patients who do not develop depression. Reserpine depletes neuronal stores of $\mathrm{NE}$ and serotonin and induces increased activity of tyrosine hydroxylase, the rate-limiting step for biosynthesis of NE. Individuals may differ in their capacity to step up NE biosynthesis or individuals with low-normal stores of NE might be more severely depleted at similar doses of reserpine. Whatever the mechanism, reserpine unmasks a predisposition to depression. It should be emphasized that so general a phenotype as depression is likely to be predisposed to or mediated by multiple mechanisms, even if various alterations in biogenic amine metabolism serve as a common pathogenetic pathway. Differential responses to therapy and differential susceptibility to precipitation of attacks with reserpine or alpha methyl dopa or ACTH may provide insights and investigational 'handles' into the heterogeneous causes of the affective disorders.

SCHIZOPHRENIA Particular phenothiazines do not appear to be relatively better than others for different clinical subtypes of schizophrenic illness (Hollister, 1970). The extrapyramidal side effects of phenothiazines, however, do occur at a lower dose and with higher incidence in patients with a family history of spontaneous Parkinson's disease (Myrianthopoulos et al., 1969). Given the evidence for genetic predisposition to schizophrenia (Rosenthal, 1970), one may wonder whether individuals who have a schizophrenic-like reaction to amphetamines or LSD are genetically pre- 
disposed to such psychotic reactions and would have been at a relatively high risk for development of 'spontaneous' schizophrenia. Pharmacological deductions have focused attention on dopamine as a possible neurotransmitter mediator of at least some types of schizophrenia. The active groups of potent antipsychotic phenothiazines, when viewed in a three-dimensional molecular model, appear to resemble the molecular conformation of dopamine (Horn and Snyder, 1971). The equiva. lence of $\mathrm{D}$ - and L-amphetamine in inducing psychosis also points to involvement of dopamine (Snyder et al., 1970; Angrist et al., 1971).

SEIZURE DISORDERS The normal pattern of electrical activity in the brain, as measured by the electroencephalogram (EEG), is determined almost entirely by multiple genetic factors (Vogel, 1970). Several single-gene mediated variants of the normal EEG have been described, affecting altogether about $15 \%$ of the general population (Vogel, 1970). The clinical significance of these variants is unknown. Studies are needed to determine whether individuals with different baseline EEG patterns have different responses to various psychopharmacological agents. The variety of anticonvulsive agents employed in clinical seizure disorders, for which biochemical mechanisms have yet to be elucidated, do not correlate very closely with clinical categories of seizure disorder. Family studies to explore pharmacogenetic relationships have not yet been performed.

DRUGS OF ABUSE AND ADDICTION SYNDROMES The most commonly abused agent, of course, is ethanol. Individuals differ markedly in tolerance for alcohol and in susceptibility to its effects. Recent studies with half siblings have shown that the excess familial incidence of alcoholism is among the biologically-related and not the adoptive family members, pointing to genetic rather than environmental influences (Schuckit, 1972). Genetic factors could play a role in variation of the acute intoxicating effects of alcohol, in the likelihood of chronic addiction, and in the risk of medical and behavioural complications (Omenn and Motulsky, 1972). Genetic factors are almost entirely responsible for individual differences in the rate of elimination of ethanol from the blood (Vesell et al., 1971). Eskimos and Indians metabolize ethanol less rapidly than do Caucasians in western Canada (Fenna et al., 1971). Most Oriental adults and infants show facial flushing and increased pulse pressure after doses of alcohol that have little or no effect on Caucasians (Wolff, 1972). The relationship between acute intoxication and chronic addiction is a central mystery in this field.

Among children born to mothers who were chronic alcoholics, a striking new syndrome has been recognized (Jones et al., 1973). These children have retarded intrauterine and post-natal physical and mental development, microcephaly, decreased width to the palpebral fissure causing the eyes to appear rounded, and variable limb and cardiac malformations. The toxic mechanism and genetic variation in susceptibility have yet to be investigated. Caucasian, Negro, and American Indian children have been recognized to be affected.

MINIMAL BRAIN DYSFUNCTION-HYPERKINETIC YOUNGSTERS These children, usually boys, have motor hyperactivity, distractibility, impulsivity, and a learning performance below objective expectations. Some of them, but clearly not the majority, respond strikingly to such stimulant drugs as amphetamine and methylphenidate. The variation in responsiveness to these drugs may reflect differences in the metabolism of the drug, in the susceptibility to the drug action at the level of some cell enzyme or cell membrane, or in the underlying processes which lead to the behavioural problems. Studies are in progress to try to differentiate these possibilities (Omenn, 1973a).

SIMPLY-INHERITED BEHAVIOURAL DISORDERS WITH SPECIAL VULNERABILITY TO DRUGS EXamples of autosomal dominant, autosomal recessive, and $X$-linked recessive diseases affecting the nervous system can be cited in which patients are usually susceptible to specific drugs (Cohen and Weber, 1972). Patients with Swedish or South African porphyria, in which there is a block in heme biosynthesis, may suffer their first attack when barbiturates, certian sulphonamides, the anti-fungal 
agent griseofulvin, and possibly anaesthetics, ethanol, or chloroquine are taken. The significance of preoperative or postoperative medications, especially 'routine sleeping pills', may be overlooked unless this diagnosis is considered in a patient with acute schizophrenic or paranoid psychosis. Acute porphyric attacks may remit with infusions of haematin (Watson et al., 1973). Patients with Huntington's chorea develop increased choreiform movements on L-dopa; it is not clear whether the L-dopa affects their psychological or intellectual status. The proposal that administration of L-dopa be used to detect asymptomatic carriers of the gene for Huntington's disease (Klawans et al., 1972) should be viewed with great caution, since the patient observes the test result, the ratc of false positives or false negatives is unknown, and it is conceivable that L-dopa might even accelerate the degenerative process. The Lesch-Nyhan syndrome, an X-linked recessive condition, is characterized by choreoathetosis and a bizarre, compulsive behaviour with self-mutilation of lips, fingers, and eyes. There is deficiency of an enzyme of purine metabolism, leading to abnormal metabolism of such agents as 6-mercaptopurine, azathioprine (Imuran), and allopurinol. Finally, several of the inborn errors of metabolism that lead to mental retardation can be ameliorated by treatment with special dietary regimens, which might be considered a particular form of interaction between genotype and exogenous agent. Thus, diets restricted in phenylalanine or galactose are effective in preventing the development of mental retardation in patients with phenylketonuria and galactosemia, respectively. When an inherited enzyme abnormality causes decreased affinity for an essential coenzyme, exogenous administration of the coenzyme in massive doses may be effective. Examples are homocystinuria and methylmalonic aciduria. The finding of rare enzyme abnormalities in co-factor binding has refuelled the suggestion that individual variability in requirements for such dietary factors might lead to dietary deficiencies on the 'usual recommended daily dose' of these vitamins (Williams, 1956). Nevertheless, there is no evidence at this point that massive doses of nicotinic acid or vitamin $\mathrm{C}$ or other common vitamins will ameliorate schizophrenia or enhance intelligence.

The examples given here of genetically-determined variation in the metabolism of certain commonly used drugs or in the risk of side-effects probably represent only the tip of the iceberg in understanding the host-drug interaction in clinical medicine. From the point of view of investigating common psychiatric syndromes, the variation in patients' responses to commonly used pharmacological agents offers one of the most promising 'handles' on differentiating multiple causes of apparently similar clinical syndromes. We may hope that such pharmacogenetic investigation will lead to more rational therapy tailored to the needs of individual patients.

GILBERT S. OMENN. AND ARNO G. MOTULSKY

The work was supported by grant GM 15253 and a Research Development Award (G.S.O.) from the U.S. Public Health Service.

\section{REFERENCES}

Alexanderson, B., and Sjöqvist, F. (1971). Individual differences in the pharmacokinetics of monomethylated tricyclic antidepressants: role of the genetic and environmental factors and clinical importance. Annals of the New York Academy of Sciences, 179, 739-751.

Angrist, B. M., Shopsin, B., and Gershon, S. (1971). The comparative psychotomimetic effects of stereoisomers of amphetamine. Nature, 234, 152-153.

Angst, J. (1964). Antidepressiver Effekt and genetische Faktoren. Arzneimittelforschung, 14, Suppl. 496-500.

Cohen, S. N., and Weber, W. W. (1972). Pharmacogenetics. Pediatric Clinics of North America, 19, 21-36.

Evans, D. A. P. (1969). Pharmacogenetics. In Selected Topics in Medical Genetics, pp. 69-109. Edited by C. A. Clarke. Oxford University Press: London.
Evans, D. A. P., Davison, K., and Pratt, R. T. C. (1965). The influence of acetylator phenotype on the effects of treating depression with phenelzine. Clinical Pharmacology and Therapeutics, 6, 430-435.

Fenna, D., Mix, L., Schaefer, O., and Gilbert, J. A. L. (1971). Ethanol metabolism in various racial groups. Canadian Medical Association Journal, 105, 472-475.

Gershon, E. S., Dunner, D. L., and Goodwin, F. K. (1971). Toward a biology of affective disorders. Genetic contributions. Archices of General Psychiatry, 25, 1-15.

Harris, T. H. (1957). Depression induced by Rauwolfia compounds. American Journal of Psychiatry, 113, 950.

Hollister, L. E. (1970). Choice of antipsychotic drugs. American Journal of Psychiatry, 127, 186-190. 
Horn. A. S., and Snyder, S. H. (197!). Chlorpromazine and dopannine: conformational similarities that correlate with the antischizophrenic activity of phenothiazine drugs. Proceedings of the National Academy of Sciences, 68, 2325-2328.

Johnstone, E. C., and Marsh, W. (1973). Acetylator status and response to phenelzine in depressed patients. Lancef I, $567-570$.

Jones, K. L., Smith, D. W., Ulleland, C. N., and Streissguth, A. P. (1973). Pattern of malformation in offspring of chronic alcoholic mothers. Lancet, 1, 1267-1271.

Kalow, W. (1962). Pharmacogenetics: Heredity and the Response to Drugs. Saunders: Philadelphia.

Klawans, H. L., Jr., Paulson, G. W., Ringel, S. P., and Barbeau, A. (1972). Use of L-dopa in the detection of presymptomatic Huntington's chorea. New England Journal of Medicine, 286, 1332-1334.

Kutt, H. (1971). Biochemical and genetic factors regulating Dilantin metabolism in man. Annals of the New York Academy of Sciences, 179, 705-722.

Morrow, A. C., and Motulsky, A. G. (1968). Rapid screening method for the common atypical pseudocholinesterase variant. Journal of Laboratory and Clinical Medicine, 71, 350-356.

Motulsky, A. G. (1972). Hemolysis in glucose-6-phosphate dehydrogenase deficiency. Federation Proceedings, 31, 1286-1292.

Motulsky, A. G. (1964). Pharmacogenetics. Progress in Medical Genetics, 3, 49-74.

Musacchio, J. M., Julou, L., Kety, S. S., and Glowinski, S. S. (1969). Increase in rat brain tyrosine hydroxylase activity produced by electroconvulsive shock. Proceedings of the National Academy of Sciences, 63, 1117-1119.

Myrianthopoulos, N. C., Waldrop, F. N., and Vincent, B. L. (1969). A repeat study of hereditary predisposition in druginduced parkinsonism. In Progress in Neuro-Genetics. Edited by A. Barbeau and J.-R. Brunette. Excerpta Medica: Amsterdam. International Congress Series No. 175, 486491.

Omenn, G. S. (1973a). Genetic issues in the syndrome of minimal brain dysfunction. Seminars in Psychiatry, 5, 5-17.
Oncmn, G. S. (19736). A pharmacogenetic approach to depression, in Bruell, J. (ed.), Prospects in Behavior Genetics. Russell Sage Foundation: New York. (In press.)

Omenn, G. S., and Motulsky, A. G. (1972). A biochemical and genetic approach to alcoholism. Annals of the New York Academy of Sciences, 197, 16-23.

Omenn, G. S., and Motulsky, A. G. (1973). Pharmacogenetics. Year Book of Drug Therapy, 1973, pp. 5-26. Year Book Medical Publishers: Chicago.

Pare, C. M. B., and Mack, J. W. (1971). Differentiation of two ganetically specific types of depression by the response to antidepressant drugs. Journal of Medical Genetics, 8, 306-309.

Pare, C. M. B., Rees, L., and Sainsbury, M. J. (1962). Differentiation of two genetically specific types of depression by the response to anti-depressants. Lancet, 2, 13401343.

Rosenthal, D. (1970). Genetic Theory and Abnormal Behavior, pp. 92-200. McGraw-Hill: New York.

Schildkraut, J. J. (1969). Neuropsychopharmacology and the affective disorders. New England Journal of Medicine, 281, 197-201, 248-255, 302-308.

Schuckit, M. A. (1972). Family history and half-sibling research in alcoholism. Annals of the New York Academy of Sciences, 197, 121-125.

Snyder, S. H., Taylor, K. M., Coyle, J. T., and Meyerhoff, J. L. (1970). The role of brain dopamine in behavioral regulation and the actions of psychotropic drugs. American Journal of Psychiatry, 127, 199-207.

Vesell, E. S., Page, J. G., and Passananti, G. T. (1971). Genetic and environmental factors affecting ethanol metabolism in man. Clinical Pharmacology and Therapeutics, 12, 192-201.

Vogel, F. (1970). The genetic basis of the normal human electroencephalogram (EEG). Humangenetik, 10, 91-114.

Watson, C. J., Dhar, G. J., Bossenmaier, I., Cardinal, R., and Petryka, Z. J. (1973). Effect of hematin in acute porphyric relapse. Annals of Internal Medicine, 79, 80-83.

Williams, R. J. (1956). Biochemical Individuality. Wiley: New York.

Wolff, P. H. (1972). Ethnic differences in alcohol sensitivity. Science, 175, 449-450. 